\title{
Cullin-associated NEDD8-dissociated protein 1, a novel interactor of rabphilin-3A, deubiquitylates rabphilin-3A and regulates arginine vasopressin secretion in PC12 cells
}

\author{
Kohtaro Nakashima ${ }^{1)}$, Seiji Takeuchi ${ }^{1)}$, Shintaro Iwama ${ }^{1)}$, Atsushi Kiyota ${ }^{1)}$, Yoshinori Yasuda ${ }^{1)}$, \\ Naoko Iwata ${ }^{1)}$, Atsushi Enomoto ${ }^{2)}$, Hiroshi Arima ${ }^{1)}$ and Yoshihisa Sugimura ${ }^{3)}$ \\ 1) Department of Endocrinology and Diabetes, Nagoya University Graduate School of Medicine, Nagoya, Aichi 466-8550, Japan \\ 2) Department of Pathology, Nagoya University Graduate School of Medicine, Nagoya, Aichi 466-8550, Japan \\ 3) Division of Endocrinology and Metabolism, Department of Internal Medicine, Fujita Health University, Toyoake, Aichi 470-1192 \\ Japan
}

\begin{abstract}
The molecular mechanism involved in the exocytosis of arginine vasopressin (AVP) is not fully known. Rabphilin-3A has been suggested as a novel autoantigen in infundibulo-neurohypophysitis (LINH), which leads to central diabetes insipidus through insufficient secretion of AVP. However, the role of rabphilin-3A in the pathogenesis of LINH remains unclear. Thus, the aim of the present study was to identify proteins binding rabphilin-3A in the posterior pituitary. Using glutathione S-transferase (GST)-pulldown assays and proteomic analyses, cullin-associated NEDD8-dissociated protein 1 (CAND1) was identified as a rabphilin-3A-binding protein in the posterior pituitary. Co-immunoprecipitation assays indicated that CAND1 interacted endogenously with rabphilin-3A. In addition, immunohistochemistry experiments showed that CAND1 immunoreactivity was detected mainly in the posterior pituitary, intermediate lobe, and the supraoptic nucleus in the hypothalamus, and less in the anterior lobe, partially co-localizing with rabphilin-3A. Overexpression of CAND1 resulted in deubiquitylation of rabphilin-3A in PC12 cells. Moreover, overexpression of CAND1 in PC12 cells co-transfected with AVP enhanced both basal and KCl-stimulated AVP secretion. The findings indicate that CAND1 inhibits the ubiquitylation of rabphilin-3A and positively regulates AVP secretion. These data shed light on a novel potential mechanism involving rabphilin-3A in AVP secretion, and suggest a new role of CAND1 as a regulator of hormone or neurotransmitter secretion.
\end{abstract}

Key words: Vasopressin, Rabphilin-3A, Cullin-associated NEDD8-dissociated protein 1, Deubiquitylation, Secretion

\begin{abstract}
ARGININE VASOPRESSIN (AVP) is a hormone secreted from the posterior pituitary through exocytosis, a process involving numerous steps [1-3]. Rabphilin-3A has been suggested as a major autoantigen in infundibuloneurohypophysitis (LINH) and autoantibodies against rabphilin-3A may be useful as diagnostic markers for LINH [4]. Rabphilin-3A is a specific GTP-Rab3A-binding protein on secretory granules $[5,6]$, regulating neurotransmitter release, and acting in conjunction with synaptosomal-associated protein 25 (SNAP25), Rab27A, and syntaxin 1 [7-11]. Although the involvement of

Submitted Sep. 11, 2017; Accepted Nov. 30, 2017 as EJ17-0399

Released online in J-STAGE as advance publication Jan. 23, 2018

Correspondence to: Yoshihisa Sugimura, Division of Endocrinology

and Metabolism, Department of Internal Medicine, Fujita Health University, 1-98 Dengakugakubo, Toyoake, Aichi 470-1192, Japan.

E-mail: sugiyosi@med.nagoya-u.ac.jp
\end{abstract}

rabphilin-3A in AVP secretion has not been previously characterized, preliminary data showed that rabphilin-3A may be involved in the docking and fusion of AVP vesicles, thereby regulating AVP release and water homeostasis in the body. However, the detailed mechanism of AVP release mediated by rabphilin-3A in the posterior pituitary is largely unknown.

The aim of this study was to identify new interactors of rabphilin-3A. CAND1 was found to interact with rabphilin-3A in the posterior pituitary, suggesting that CAND1 may deubiquitylate rabphilin-3A and mediate secretion in endocrine cells.

\section{Materials and Methods}

\section{Animal model}

In this study, Sprague-Dawley rats (sex: male; weight: 
Table 1 List of primary antibodies and working dilutions

\begin{tabular}{|c|c|c|c|c|c|c|c|}
\hline Protein & Catalog No & Poly/Mono & Host & IB & $\mathrm{IHC}$ & IP & Company \\
\hline \multirow{5}{*}{ Rabphilin-3A } & ab59259 & Poly & Rabbit & $1: 500$ & & & Abcam \\
\hline & AP06576PU-N & Poly & Rabbit & $1: 500$ & & & Acris \\
\hline & ab68857 & Poly & Rabbit & & $1: 150$ & & Abcam \\
\hline & RM3701 & Mono & Mouse & & & $10 \mu \mathrm{g}$ & ECM Biosciences \\
\hline & sc-366358 & Poly & Rabbit & & & $10 \mu \mathrm{g}$ & Santa Cruz Biotechnology \\
\hline CAND1 & sc-10672 & Poly & Goat & $1: 100$ & $1: 50$ & $10 \mu \mathrm{g}$ & Santa Cruz Biotechnology \\
\hline \multirow[t]{2}{*}{ SNAP25 } & ab5666 & Poly & Rabbit & $1: 1,000$ & & & Abcam \\
\hline & 111011 & Mono & Mouse & & & $10 \mu \mathrm{g}$ & Synaptic Systems \\
\hline Mouse IgG & sc-2025 & Mono & Mouse & & & $10 \mu \mathrm{g}$ & Santa Cruz Biotechnology \\
\hline Goat IgG & pp40 & Poly & Goat & & & $10 \mu \mathrm{g}$ & Chemicon \\
\hline Rabbit IgG & sc-2027 & Mono & Rabbit & & & $10 \mu \mathrm{g}$ & Santa Cruz Biotechnology \\
\hline Ubiquitin (P4D1) & sc-8017 & Mono & Mouse & $1: 1,000$ & & & Santa Cruz Biotechnology \\
\hline$\beta$-actin & A2228 & Mono & Mouse & $1: 2,000$ & & & Sigma \\
\hline
\end{tabular}

Poly/Mono, polyclonal or monoclonal antibody; IB, dilutions used for immunoblotting; IHC, dilutions used for immunohistochemistry; ICC, dilutions used for immunocytochemistry; IP, total protein used for immunoprecipitation.

250-300 g) (Chubu Science Materials, Nagoya, Japan) were used. The animals were kept under controlled conditions (temperature: $23.0 \pm 0.5^{\circ} \mathrm{C}$; lights: 09:00-21:00 hours) and fed with standard rat chow. The procedures/ protocols followed the institutional guidelines for animal care at Nagoya University.

\section{Plasmid constructs}

Rabphilin-3A (clone ID AK730001I15) (DNAFORM, Yokohama, Japan) and CAND1 cDNA (clone ID FXC01125) (Promega, Madison, WI, USA) were purchased prior to initiation of experiments.

\section{Antibodies}

Primary antibodies utilized in these experiments are presented in Table 1.

\section{Recombinant proteins}

The Gateway system (Invitrogen, Carlsbad, CA, USA) was used to construct plasmids for GST-rabphilin-3A. The cDNA fragment was sub-cloned into the pENTR-DTOPO vector, followed by recombination into the destination vector (pCAGGS-FLAG, kindly provided by Dr. Watanabe [Nagoya University]) with LR clonase II (Invitrogen). Escherichia coli (Rosetta DE3) cells were used to express GST-rabphilin-3A. GST-rabphilin-3A was purified by Glutathione Sepharose 4B chromatography
(GE Healthcare UK Ltd., Hertfordshire, UK). Dr. K. Kaibuchi (Nagoya University) provided the GST-cde42GTP $\gamma$ S.

\section{Posterior pituitary lysates}

Posterior pituitaries were solubilized in lysis buffer $(500 \mu \mathrm{L})(10 \mathrm{mM}$ HEPES-NaOH [pH 7.4], 0.5\% NP40, $1 \mathrm{mM} \mathrm{CaCl}_{2}, 50 \mathrm{mM} \mathrm{NaCl}, 5 \mathrm{mM} \mathrm{MgCl}_{2}$ ) and subsequently homogenized, sonicated and centrifuged $(43,000$ rpm for $30 \mathrm{~min}$ at $4^{\circ} \mathrm{C}$ ) to collect the supernatant.

\section{In vitro binding assays}

Glutathione Sepharose 4B $\left(1 \mathrm{~h}\right.$ at $\left.4^{\circ} \mathrm{C}\right)$ was used to preclear posterior pituitary lysates. Subsequently, lysates were mixed with GST-rabphilin-3A or GST-cdc42GTP $\gamma$ S and Glutathione Sepharose 4B (overnight at $\left.4^{\circ} \mathrm{C}\right)$. The sediment was isolated and washed $(\times 4)$ with buffer (10 mM HEPES-NaOH [pH 7.4], $1 \mathrm{mM} \mathrm{CaCl}_{2}, 50$ $\mathrm{mM} \mathrm{NaCl}$, and $5 \mathrm{mM} \mathrm{MgCl}_{2}$ ). SDS sample buffer was used to elute bound proteins [12].

\section{In-gel digestion and mass spectrometry}

SDS-PAGE was used to resolve eluted samples. 2DSilver Stain-II (Cosmo Bio Co., Ltd., Tokyo, Japan) was used for silver staining. Subsequently, in-gel digestion and nano-LC-TOF MS/MS analysis was performed using the gel bands (Medical \& Biological Laboratories Co., 
Nagoya, Japan). The ProteinPilot ${ }^{\mathrm{TM}}$ (AB SCIEX, Framingham, MA, USA) was employed to search the Swiss-Prot database.

\section{Immunohistochemistry}

Transcardial perfusion with $4 \%$ paraformaldehyde phosphate-buffered saline (PBS) was applied to sacrifice the experimental animals under deep ether anesthesia. Cryostat sections $(7 \mu \mathrm{m})$ were produced by removing the brains of the animals and postfixing them in $4 \%$ paraformaldehyde PBS [13]. Subsequent steps included incubation of sections in $0.3 \%$ Triton X-100 (15 min), treatment with $50 \mathrm{mM}$ glycine PBS (15 min), blocking with 5\% bovine serum albumin $(1 \mathrm{~h})$, and incubation with primary antibodies (overnight). Subsequently, the sections were washed with PBS $(\times 3)$, and incubated with 4',6diamidino-2-phenylindole (Dojindo Laboratories, Kumamoto, Japan) and the Alexa Fluor ${ }^{\circledR}$ 488- and 594-conjugated anti-IgG secondary antibodies $(1: 1,000$ dilution) (Invitrogen) ( $1 \mathrm{~h}$ at room temperature). Following further washes with PBS $(\times 3)$, the liquid mountant anti-fade solution (ProLong ${ }^{\circledR}$ Gold, Life Technologies, Foster City, CA, USA) was applied to the sections. An inverted confocal laser scanning microscope (A1RSi; Nikon, Tokyo, Japan) or fluorescence microscope (BZ-8000; Keyence, Osaka, Japan) were used to assess fluorescently stained sections.

\section{Western blotting}

One-dimensional SDS-PAGE was performed. Nitrocellulose membranes containing the proteins were blocked in $4 \%$ milk and incubated overnight with primary antibodies. Anti-mouse or anti-rabbit IgGs (1:1,000 dilution) horseradish peroxidase (HRP)-conjugated antibodies were used as secondary antibodies $(1 \mathrm{~h})$. Membranes were subsequently incubated with the ECL ${ }^{\mathrm{TM}}$ Western Blotting Detection reagent (GE Healthcare) and visualized using X-ray film (Cosmo Bio Co., Ltd.).

\section{Quantitative RT-PCR}

The organs of the sacrificed rats were excised and stored at $-80^{\circ} \mathrm{C}$. The RNeasy Mini Kit (QIAGEN, Valencia, CA, USA) was used to extract total RNA. The PrimeScript RT Master Mix (Takara Bio Inc., Otsu, Japan) was used to reverse-transcribe $1 \mu \mathrm{g}$ of RNA (final volume: $25 \mu \mathrm{L}$ ). The SYBR Premix Ex Taq II (Takara Bio, Inc.) and a StepOne ${ }^{\mathrm{TM}}$ Real-Time PCR System (Applied Biosystems) were used to perform real-time PCR. Primers: CAND1 forward (5'-ACAGAGGTTGGACCGGC
TTG-3') and reverse (5'-GGAATGGTGAGCAGTGCA GCTA-3') (Takara Bio Inc.). The Ct-method was used to compare mRNA levels normalized to $\beta$-actin levels.

\section{Co-immunoprecipitation assays}

G Sepharose (GE Healthcare) $\left(1 \mathrm{~h}\right.$ at $\left.4^{\circ} \mathrm{C}\right)$ was used to preclear posterior pituitary lysates. Subsequently, lysates were incubated with $10 \mu \mathrm{g}$ of control IgG or IP antibodies at $4^{\circ} \mathrm{C}$ and incubated with $\mathrm{G}$ Sepharose $\left(3 \mathrm{~h}\right.$ at $\left.4^{\circ} \mathrm{C}\right)$. The sediments were washed $(\times 4)$ with washing buffer and proteins were eluted in SDS buffer.

\section{Cell culture}

Dulbecco's modified Eagle's medium (Life Technologies) containing $10 \%$ fetal bovine serum (Life Technologies), $10 \%$ horse serum (Life Technologies), $100 \mathrm{U} / \mathrm{mL}$ penicillin and $100 \mu \mathrm{g} / \mathrm{mL}$ streptomycin (Sigma) was used to culture $\mathrm{PC} 12$ cells at $37^{\circ} \mathrm{C}$ and $5 \% \mathrm{CO}_{2}$. Cells reaching $70-80 \%$ confluence were passaged. For transfection, PC12 cells were plated onto $35-\mathrm{mm}$ or $100-\mathrm{mm}$ dishes until they reached $60-70 \%$ confluence.

\section{Overexpression of CAND1}

PC12 cells were transfected with the mouse CAND1 vector or with an empty vector using the Lipofectamine1 3000 Reagent (Life Technologies). Immunoblotting was used to assess overexpression in lysates $48 \mathrm{~h}$ after transfection.

\section{Analysis of AVP release}

AVP assays were carried out $48 \mathrm{~h}$ after transfection. PC12 cells were washed once with PBS at $37^{\circ} \mathrm{C}$ and subsequently incubated with either a low $\mathrm{K}^{+}$solution (137 $\mathrm{mM} \mathrm{NaCl}, 5 \mathrm{mM} \mathrm{KCl}, 5 \mathrm{mM}$ glucose, $1 \mathrm{mM} \mathrm{CaCl}_{2}, 1$ $\mathrm{mM} \mathrm{MgCl} 2,20 \mathrm{mM}$ HEPES, $\mathrm{pH}$ 7.4) to measure basal AVP release or a high $\mathrm{K}^{+}$solution $(87 \mathrm{mM} \mathrm{NaCl}, 55 \mathrm{mM}$

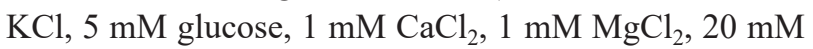
HEPES, pH 7.4) $\left(10 \mathrm{~min}\right.$ at $\left.37^{\circ} \mathrm{C}\right)$ to measure depolarization-induced AVP release. The supernatant was kept on ice. Cells were lysed in buffer $(0.5 \%$ Triton $\mathrm{X}-100,150 \mathrm{mM} \mathrm{NaCl}, 50 \mathrm{mM}$ Tris-HCl, $\mathrm{pH}$ 7.5) for 15 min at room temperature [14]. Secreted AVP and AVP retained in the cells were determined using a radioimmunoassay (RIA) kit (AVP-RIA kit YAMASA; SRL, Tokyo, Japan). Released AVP was expressed as AVP secreted in the incubation solution divided by total AVP.

\section{Statistical analyses}

Student's $t$-test or one-way ANOVA followed by the 
Bonferroni test were performed. Data are presented as means \pm SEMs. $p$ values $<0.05$ denoted statistical significance.

\section{Results}

\section{Identification of CAND1 as a rabphilin-3A-binding protein in the posterior pituitary}

Glutathione S-transferase (GST) pulldown assays using GST-rabphilin-3A and rat posterior pituitary lysates were performed to identify rabphilin-3A-binding proteins. Sodium dodecyl sulphate polyacrylamide gel electrophoresis (SDS-PAGE) was utilized followed by silver staining. A band migrating band (approximately $120 \mathrm{kDa}$ ) was detected (boxed and indicated with the arrow in Fig. 1a). However, this band was not present in the control samples using GST-cdc42-GTP $\gamma \mathrm{S}$ beads (Fig. 1a). This finding excluded the possibility of nonspecific binding. In-gel digestion and nano-liquid chromatography-mass spectrometry/mass spectrometry (LC/MS/MS) analysis were used to examine this band. The presence of cullin-associated NEDD8-dissociated protein 1 (CAND1) was confirmed and its expression was determined by Western blotting using anti-CAND1 antibodies. The presence of CAND1 in the pull-down sample was confirmed (Fig. 1b). These findings corroborated those of the proteomic analyses. Furthermore, the presence of CAND1 in the posterior pituitary was confirmed by Western blotting (Fig. 1b).

Co-immunoprecipitation assays using posterior pituitary lysates were performed to investigate endogenous interactions between CAND1 and rabphilin-3A. Rabphilin-3A was shown to co-immunoprecipitate with CAND1 (Fig. 1c). In addition, these experiments showed that SNAP25 co-immunoprecipitated with rabphilin-3A, but not with CAND1 (Fig. 1d).

\section{CAND1 is expressed in the pituitary and AVP neurons in the hypothalamus}

CAND1 was found to be mainly localized in the posterior pituitary and intermediate lobe, as indicated by immunolocalization analyses (Fig. 2a, b). Western blotting confirmed the expression of CAND1 (Fig. 1b). The immunoreactivity of CAND1 in the anterior pituitary was weak compared with those in the posterior pituitary and intermediate lobe (Fig. 2a). In addition, CAND1 was detected in the supraoptic nucleus (SON) in the hypothalamus (Fig. 2c, d). The immunoreactivity of CAND1 partially overlapped with that of rabphilin-3A in the pos- terior pituitary and SON (Fig. 2a-d). CAND1 immunoreactivity was also detected in the PVN and partially overlapped with that of rabphilin-3A (data not shown). Subsequently, we evaluated mRNA levels by reverse transcription polymerase chain reaction (RT-PCR) analysis. CAND1 mRNA levels in the rat hypothalamus were comparable to those observed in the rat cortex and liver (Fig. 2e).

\section{CAND1 deubiquitylates rabphilin-3A in PC12 cells}

CAND1 regulates the formation of the SCF (SKP1, CUL1, F-box proteins) ubiquitin ligase complex, and its binding to the complex results in reduced substrate ubiquitylation $[15,16]$. Overexpression experiments using plasmid constructs were performed in $\mathrm{PC} 12$ cells to investigate the effects of CAND1 on rabphilin-3A ubiquitylation. Rabphilin-3A was immunoprecipitated and ubiquitylation was detected using anti-ubiquitin antibodies in the absence or presence of $10 \mu \mathrm{M}$ MG132 (a proteasome inhibitor). Similarly, rabphilin-3A was immunoprecipitated from control, CAND1-overexpressing and MG132-treated groups (Fig. 3a). As expected, the levels of ubiquitylated rabphilin-3A were decreased in the CAND1-overexpressing group and increased in the MG132-treated group compared with the control group (Fig. 3b, c). Expression of rabphilin-3A was increased in the CAND1-overexpressing cells compared with the control cells (Fig. 3d, e). These data indicate that CAND1 deubiquitylates rabphilin-3A and prevents its degradation in PC12 cells.

\section{Overexpression of CAND1 in PC12 cells co-transfected with AVP enhances both basal and KCl-stimulated AVP secretion}

Overexpression experiments using plasmid constructs and measurements of AVP levels in growth media and lysates of PC12 cultures were performed to investigate the role of CAND1 in AVP secretion. Overexpression of CAND1 was confirmed by immunoblotting (Fig. 4a). Overexpression of CAND1 in PC12 cells co-transfected with AVP enhanced both basal and KCl-stimulated AVP secretion $(p<0.05)$ (Fig. 4b).

\section{Discussion}

In this study, CAND1 was identified as an interactor of rabphilin-3A in the posterior pituitary, using GSTpulldown assays and proteomic analyses. CAND1 is expressed in the pituitary and AVP neurons in the hypo- 
a

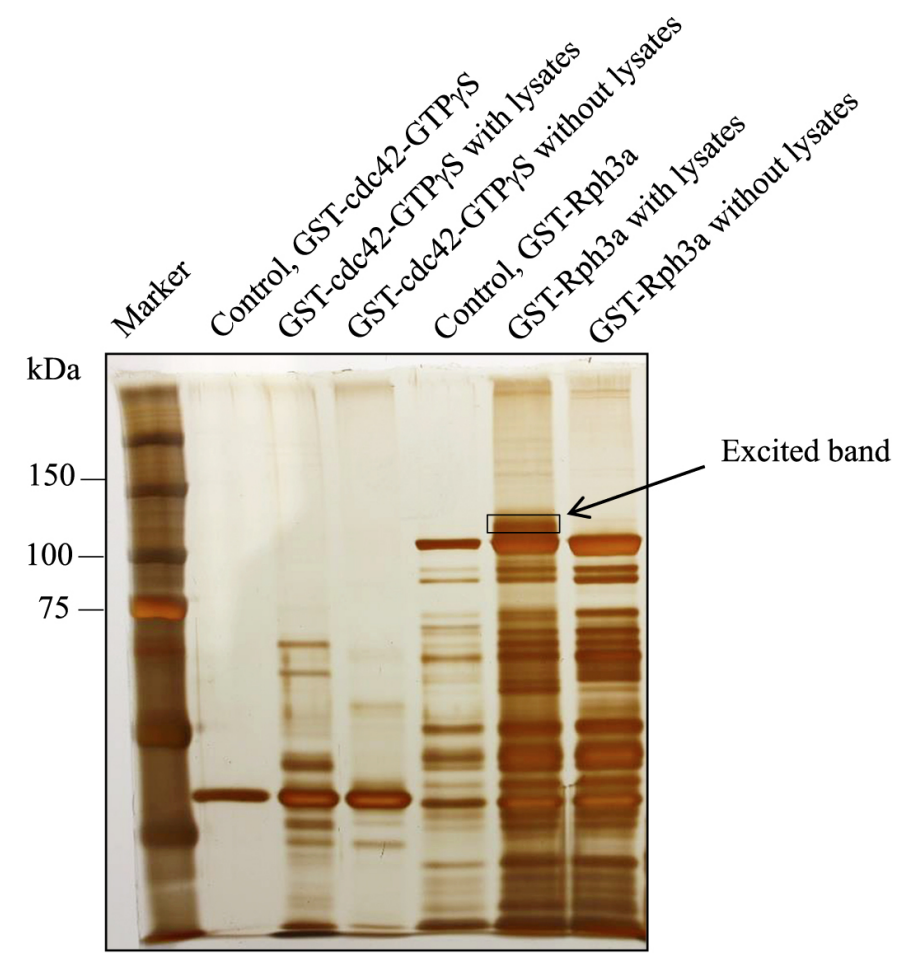

b

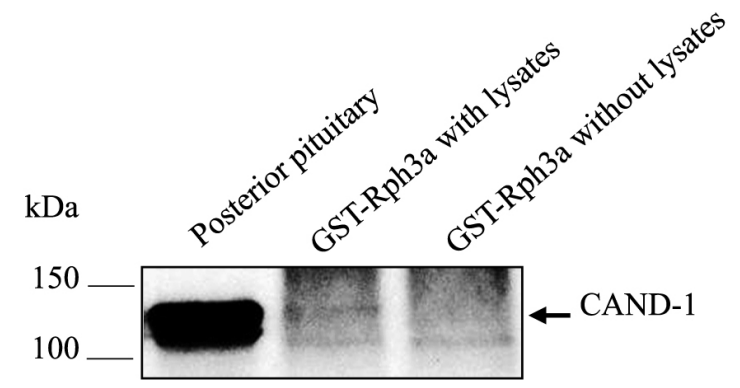

c

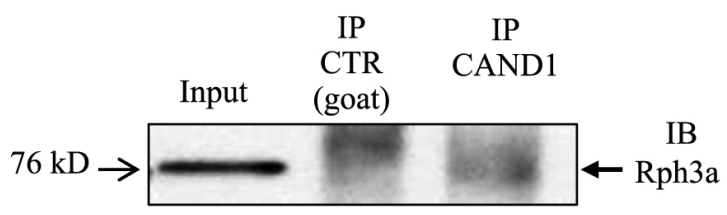

d

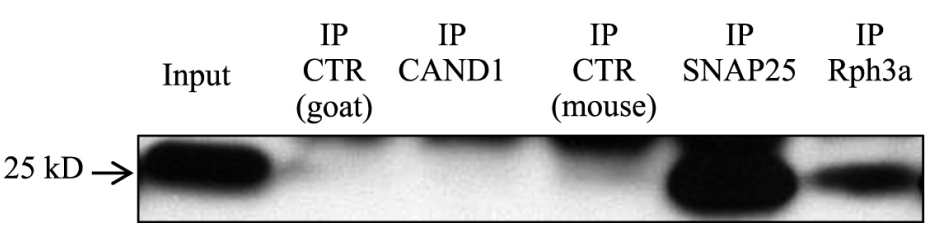

Fig. 1 Identification of CAND1 as a rabphilin-3A-binding protein in the posterior pituitary

(a) Pulldown assay. Posterior pituitary lysates incubated with GST-rabphilin-3A and glutathione (GSH) Sepharose or GST-cdc42GTP $\gamma$ S and GST Sepharose. Proteins were separated by SDS-PAGE and silver-stained. The arrow shows the band of interest. (b) Western blotting. The band detected using the anti-CAND1 antibody (arrowhead). (c, d) Co-immunoprecipitation assays with G Sepharose and goat immunoprecipitation (IP) antibodies (c), G Sepharose and goat IP antibodies or ImmunocruzE ${ }^{\mathrm{TM}}$ and mouse IP antibodies (d). SDS-PAGE followed by Western blotting using anti-rabphilin-3A (c) or anti-SNAP25 antibodies (d). Input: 1/200 (volume) of the total lysates. CTR: IP with anti-IgG antibody. Rph3a; rabphilin-3A 

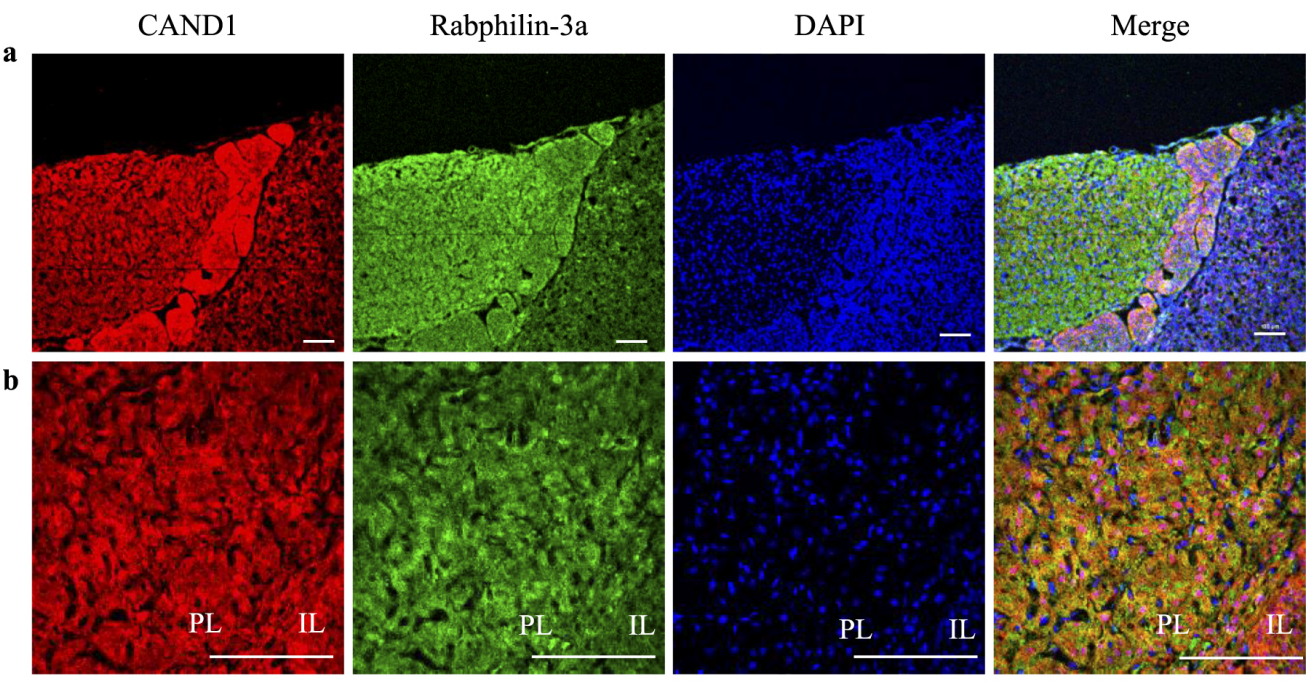

c
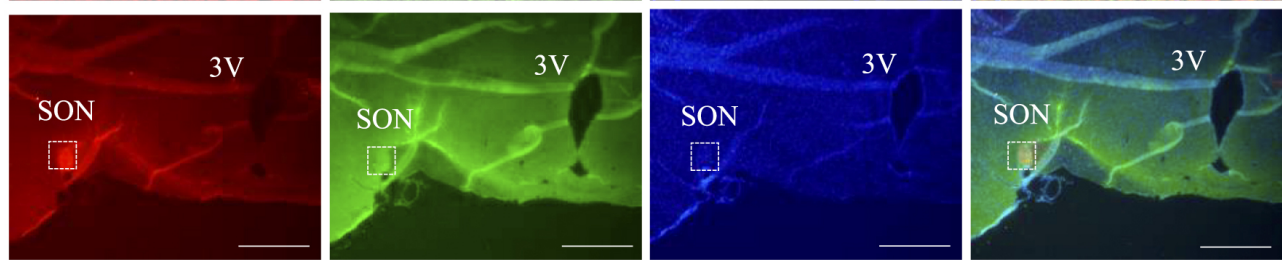

d
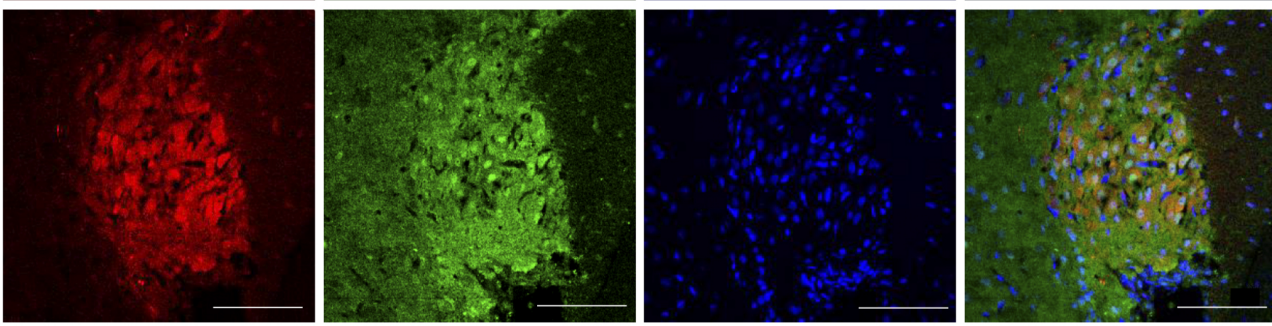

e

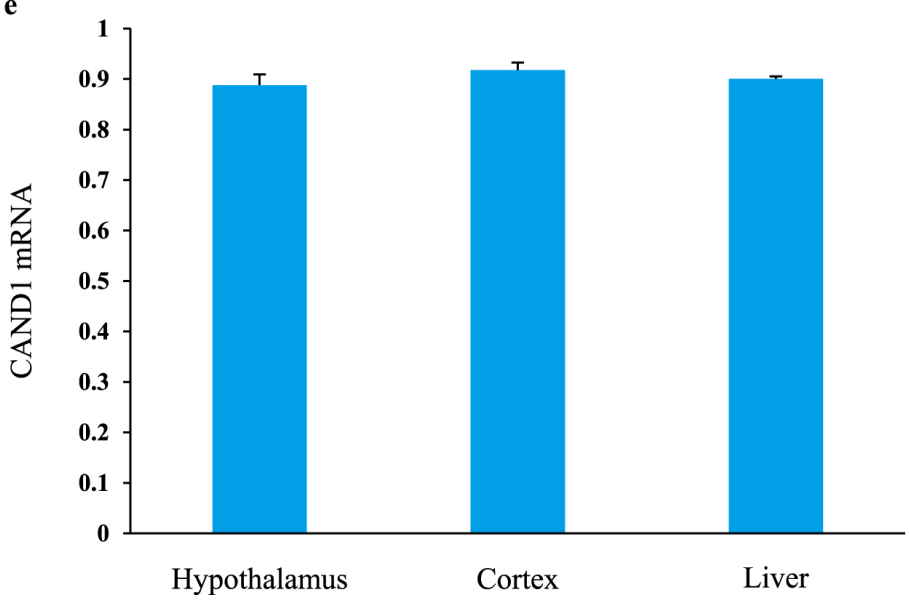

Fig. 2 CAND1 localizes in the hypothalamo-neurohypophysial system

(a-d) Cryosections were double-stained with anti-CAND1 and anti-rabphilin-3A. 4',6-diamidino-2-phenylindole was used to stain nuclei. The panels on the right show merged images. (b, d) Higher magnification of a and c, respectively. Scale bars correspond to $100 \mu \mathrm{m}$. AL: anterior lobe of the pituitary; IL: intermediate lobe; PL: posterior lobe; SON: supraoptic nuclei. (e) Levels of CAND1 mRNA in the rat hypothalamus, cortex and liver using quantitative RT-PCR. Data were normalized to $\beta$-actin and presented as means \pm SEMs. $(n=2)$. 
a

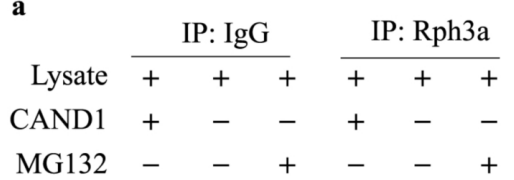

b
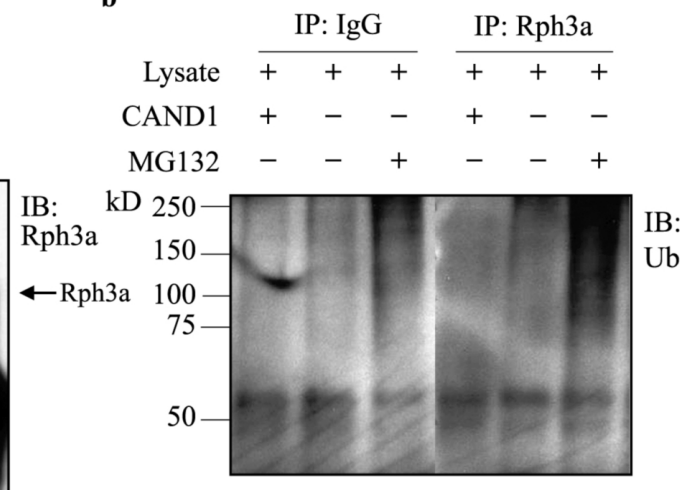

c

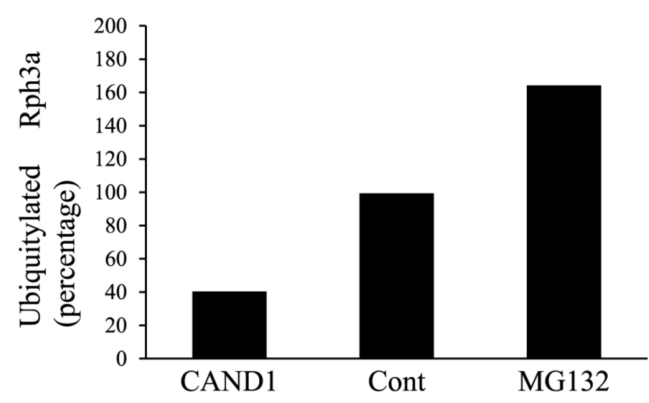

d

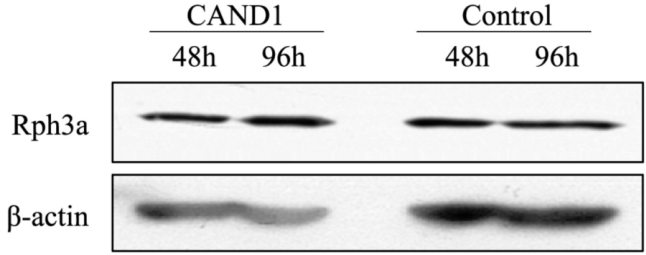

$\mathbf{e}$

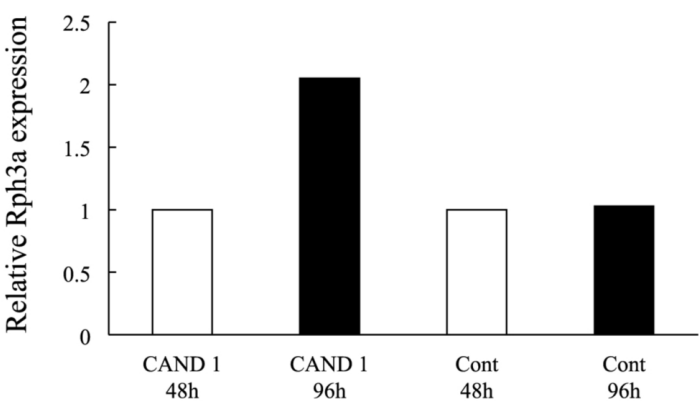

Fig. 3 CAND1 deubiquitylates rabphilin-3A in PC12 cells

PC12 cells were transfected with an empty vector (control) or a CAND1 vector (CAND1). (a, b) Representative images. After 48 $\mathrm{h}$, extracts of PC12 cells treated $\pm 10 \mu \mathrm{M}$ MG132 (4 h) were immunoprecipitated with anti-rabphilin-3A antibodies, resolved by SDS-PAGE and blotted with anti-rabphilin-3A (a) or anti-ubiquitin antibodies (b). (c) Quantification of the relative intensities of ubiquitylated rabphilin-3A signals. The experiment was performed in duplicate. (d) After 48 and $96 \mathrm{~h}$, the expression of rabphilin-3A was examined by Western blotting. (e) Quantification of the relative expression of rabphilin-3A. Data were normalized to $\beta$-actin levels. The experiment was performed in duplicate. CAND1: CAND1 overexpression without MG132; Cont: transfection with empty vector, without MG132; MG132: transfection with empty vector, with MG132. Rph3a; rabphilin-3A 
$\mathbf{a}$

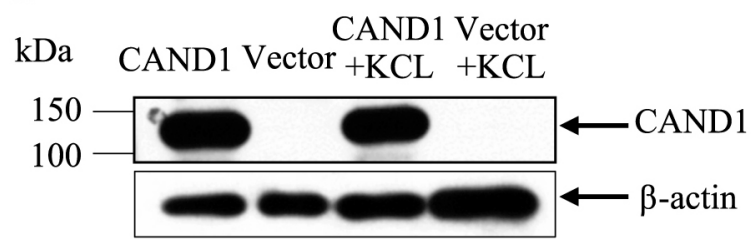

b

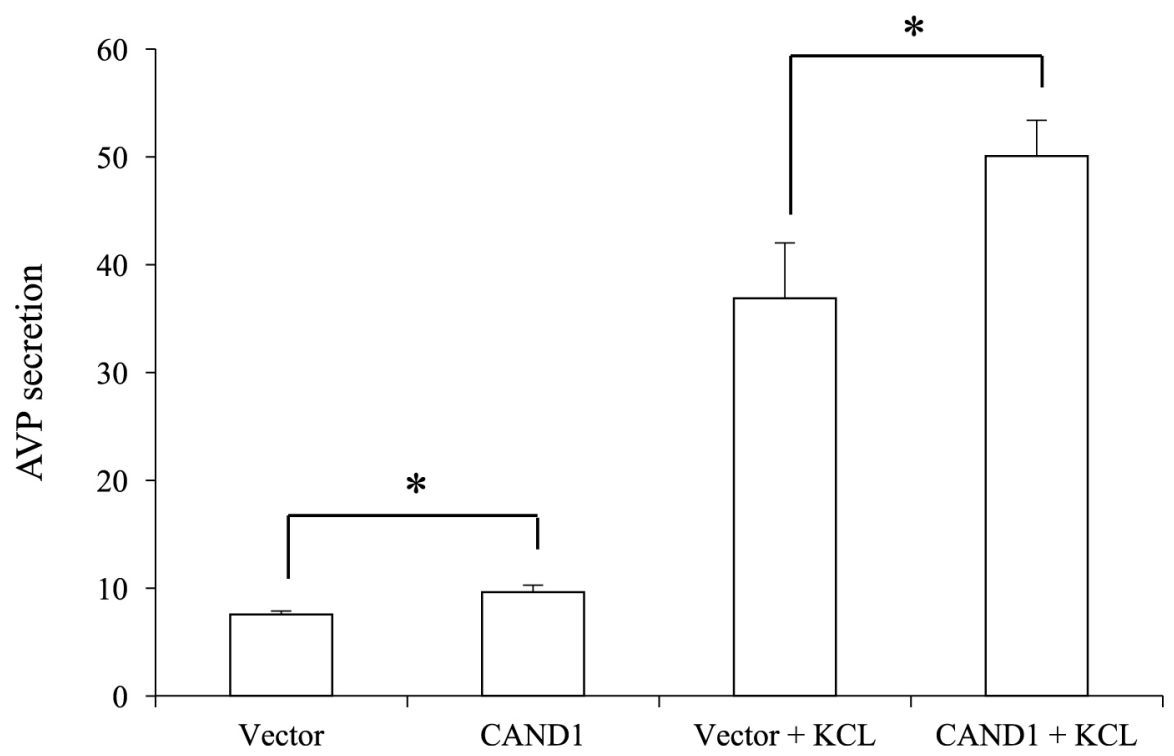

Fig. 4 Overexpression of CAND1 in PC12 cells co-transfected with AVP enhances AVP secretion

(a) Overexpression of CAND1. PC12 cells were transfected with an empty vector (control) or a CAND1 vector. After 48 h, CAND1 levels were examined by Western blotting using anti-CAND1 antibodies (CAND1). Data were normalized to $\beta$-actin. (b) The AVP release assay was carried out $48 \mathrm{~h}$ after transfection. Empty vector: $n=11$; CAND1 vector: $n=12$; empty vector with $\mathrm{KCl}: n=11$; CAND1 vector with $\mathrm{KCl}: n=12$. The final $\mathrm{KCl}$ concentration was $100 \mathrm{mM}$. Data are presented as means $\pm \mathrm{SEMs} . * p$ $<0.05$.

thalamus. The present data indicate that CAND1 deubiquitylates rabphilin-3A and regulates secretion of AVP in PC12 cells.

Immunofluorescence analyses revealed the presence of CAND1 mainly in the posterior pituitary, the internal lobe and the SON of the hypothalamus, where AVP neurons are present. It has been previously reported that rabphilin-3A is expressed in the posterior pituitary and AVP neurons in the hypothalamus [4], where CAND1 and rabphilin-3A co-localized in our experiments. These results suggest that CAND1 associates with rabphilin-3A and plays a role in AVP secretion.

Rabphilin-3A is expressed in neurons and neuroendocrine cells, where it co-localizes with Rab3A or Rab27A on secretory vesicle membranes [17]. Rabphilin-3A is a protein that binds the active form of Rab3A, which controls $\mathrm{Ca}^{2+}$-triggered exocytosis. Although rabphilin-3A overexpression enhances regulated secretion in chromaffin and PC12 cells [18, 19], the exact role of rabphilin-3A in exocytosis remains under debate. It has also been reported that rabphilin-3A mutants that do not bind Rab3 can stimulate secretion as effectively as wild type PC12 and insulin-secreting cells [20]. The effects of rabphilin-3A on secretion are thus likely to be mediated through interactions with unknown partners [20].

PC12 cells were used as a model to investigate the function of CAND1 in AVP secretion. Overexpression of CAND1 in cells co-transfected with AVP significantly 
increased AVP secretion both in the presence or absence of KCl-stimulated AVP secretion, indicating that CAND1 regulates both basal and depolarization-evoked AVP secretion in PC12 cells. This is the first report showing an involvement of CAND1 in the secretion of hormones or neurotransmitters.

CAND1 was isolated as a cullin-1 (CUL1)-binding protein. CUL1 is an essential component of the SCF ubiquitin E3 ligase complex. CAND1 binds to unneddylated CUL1 and regulates the formation of the SCF ubiquitin E3 ligase complex, thus regulating ubiquitindependent proteolysis of many regulatory proteins $[15$, 21, 22]. It is also known that CAND1 modulates SCF complexes [16, 23-25]. The present data suggest that CAND1 deubiquitylates rabphilin-3A. Ubiquitylation influences many biological processes, including degradation via the proteasome, protein localization, and regulation of activity [26, 27]. Inhibition of rabphilin-3A degradation by CAND1 may be one of the possible effects of CAND1 on hormone secretion. Since rabphilin-3A may regulate AVP release in vivo (unpublished data), the inhibition of rabphilin-3A degradation mediated by CAND1 may positively regulate AVP secretion.

The ubiquitin-proteasome system (UPS) modulates the levels of synaptic proteins, such as synaptophysin, syntaxin, Munc1 and Rab3-interacting molecule 1 (RIM1), and Bassoon and Piccolo in the presynaptic active zone [28-33]. Yao et al. reported that SCRAPPER, an E3 ubiquitin ligase, directly binds to and ubiquitylates RIM1 regulating synaptic vesicle release via RIM1 degradation and proteasome activity in vivo [32]. Rab proteins play a central role in vesicle transport and Rab11A has been reported to be ubiquitylated and activated by HACE1, a HECT domain and Ankyrin repeat containing E3 ubiq- uitin ligase conjugated with the $\beta 2$-adrenergic receptor [34].

Further investigation is warranted to determine the precise role of CAND1. For instance, it may be important to determine the rabphilin-3A site for CAND1 interaction and whether or not this interaction is responsible for the involvement of CAND1 in secretion. In addition, the role of CAND1 in AVP secretion and water homeostasis in vivo ought to be clarified. Such studies could include the investigation of changes in expression and interaction of CAND1 and rabphilin-3A which may be induced by the salt loading or water deprivation test, ideally using knockout mice. The etiology of CDI is undetermined in approximately $50 \%$ of patients suffering from this condition [35]. Future efforts may be aimed at screening patients with idiopathic CDI for mutations in the rabphilin-3A and CAND1 genes.

\section{Acknowledgements}

This study was funded through grants-in-aid for scientific research awarded to Y.S. by the Japanese Society for the Promotion of Science 24591360 and Research on Hypothalamo-hypophyseal Disorders from the Ministry of Health, Labour and Welfare, Japan.

\section{Disclosure}

The authors declare no competing financial interests.

\section{Author contributions}

K.N., A.E., and Y.S. designed experiments. K.N., S.T., S.I., A.K., Y.Y., N.I., A.E., M.T., H.A., and Y.S. performed experiments. K.N. and Y.S. analyzed data. K.N. and Y.S. wrote the paper.

\section{References}

1. Burbach JP, Luckman SM, Murphy D, Gainer H (2001) Gene regulation in the magnocellular hypothalamoneurohypophysial system. Physiol Rev 81: 1197-1267.

2. Tobin V, Leng G, Ludwig M (2012) The involvement of actin, calcium channels and exocytosis proteins in somatodendritic oxytocin and vasopressin release. Front Physiol 3: 261 .

3. Gainer H, Chin H (1998) Molecular diversity in neurosecretion: reflections on the hypothalamo-neurohypophysial system. Cell Mol Neurobiol 18: 211-230.

4. Iwama S, Sugimura Y, Kiyota A, Kato T, Enomoto A, et al. (2015) Rabphilin-3A as a targeted autoantigen in lymphocytic infundibulo-neurohypophysitis. J Clin Endocrinol Metab 100: E946-E954.

5. Shirataki H, Kaibuchi K, Yamaguchi T, Wada K, Horiuchi $\mathrm{H}$, et al. (1992) A possible target protein for smg-25A/ rab3A small GTP-binding protein. J Biol Chem 267: 10946-10949.

6. Shirataki H, Kaibuchi K, Sakoda T, Kishida S, Yamaguchi T, et al. (1993) Rabphilin-3A, a putative target protein for smg p25A/rab3A p25 small GTP-binding protein related to synaptotagmin. Mol Cell Biol 13: 2061-2068. 
7. Deak F, Shin $\mathrm{OH}$, Tang J, Hanson $\mathrm{P}$, Ubach J, et al. (2006) Rabphilin regulates SNARE-dependent re-priming of synaptic vesicles for fusion. EMBO J 25: 2856-2866.

8. Tsuboi T, Fukuda M (2005) The C2B domain of rabphilin directly interacts with SNAP-25 and regulates the docking step of dense core vesicle exocytosis in PC12 cells. J Biol Chem 280: 39253-39259.

9. McKiernan CJ, Stabila PF, Macara IG (1996) Role of the Rab3A-binding domain in targeting of rabphilin-3A to vesicle membranes of PC12 cells. Mol Cell Biol 16: 49854995.

10. Tsuboi T, Kitaguchi T, Karasawa S, Fukuda M, Miyawaki A (2010) Age-dependent preferential dense-core vesicle exocytosis in neuroendocrine cells revealed by newly developed monomeric fluorescent timer protein. Mol Biol Cell 21: 87-94.

11. Ferrer-Orta C, Perez-Sanchez MD, Coronado-Parra T, Silva C, Lopez-Martinez D, et al. (2017) Structural characterization of the Rabphilin-3A-SNAP25 interaction. Proc Natl Acad Sci USA 114: E5343-E5351.

12. Takeuchi S, Iwama S, Takagi H, Kiyota A, Nakashima K, et al. (2016) Tomosyn negatively regulates arginine vasopressin secretion in embryonic stem cell-derived neurons. PloS One 11: e0164544.

13. Sugimura Y, Murase T, Takefuji S, Hayasaka S, Takagishi $\mathrm{Y}$, et al. (2005) Protective effect of dexamethasone on osmotic-induced demyelination in rats. Exp Neurol 192: 178-183.

14. Machado HB, Liu W, Vician LJ, Herschman HR (2004) Synaptotagmin IV overexpression inhibits depolarizationinduced exocytosis in PC12 cells. J Neurosci Res 76: 334 341.

15. Zheng J, Yang X, Harrell JM, Ryzhikov S, Shim EH, et al. (2002) CAND1 binds to unneddylated CUL1 and regulates the formation of SCF ubiquitin E3 ligase complex. Mol Cell 10: 1519-1526.

16. Flick K, Kaiser P (2013) Set them free: F-box protein exchange by Cand1. Cell Res 23: 870-871.

17. Mizoguchi A, Yano Y, Hamaguchi H, Yanagida H, Ide C, et al. (1994) Localization of rabphilin-3A on the synaptic vesicle. Biochem Biophys Res Commun 202: 1235-1243.

18. Chung SH, Takai Y, Holz RW (1995) Evidence that the Rab3a-binding protein, rabphilin3a, enhances regulated secretion. Studies in adrenal chromaffin cells. J Biol Chem 270: 16714-16718.

19. Komuro R, Sasaki T, Orita S, Maeda M, Takai Y (1996) Involvement of rabphilin-3A in $\mathrm{Ca} 2+$-dependent exocytosis from PC12 cells. Biochem Biophys Res Commun 219: 435-440.

20. Joberty G, Stabila PF, Coppola T, Macara IG, Regazzi R (1999) High affinity Rab3 binding is dispensable for rabphilin-dependent potentiation of stimulated secretion. $J$ Cell Sci 112: 3579-3587.
21. Liu J, Furukawa M, Matsumoto T, Xiong Y (2002) NEDD8 modification of CUL1 dissociates p120 (CAND1), an inhibitor of CUL1-SKP1 binding and SCF ligases. Mol Cell 10: 1511-1518.

22. Bornstein G, Ganoth D, Hershko A (2006) Regulation of neddylation and deneddylation of cullin1 in SCFSkp2 ubiquitin ligase by F-box protein and substrate. Proc Natl Acad Sci USA 103: 11515-11520.

23. Pierce NW, Lee JE, Liu X, Sweredoski MJ, Graham RL, et al. (2013) Cand1 promotes assembly of new SCF complexes through dynamic exchange of $\mathrm{F}$ box proteins. Cell 153: 206-215.

24. Olma MH, Dikic I (2013) Cullins getting undressed by the protein exchange factor Cand1. Cell 153: 14-16.

25. Wu S, Zhu W, Nhan T, Toth JI, Petroski MD, et al. (2013) CAND1 controls in vivo dynamics of the cullin 1-RING ubiquitin ligase repertoire. Nat Commun 4: 1642.

26. Raiborg C, Stenmark H (2009) The ESCRT machinery in endosomal sorting of ubiquitylated membrane proteins. Nature 458: 445-452.

27. Bhoj VG, Chen ZJ (2009) Ubiquitylation in innate and adaptive immunity. Nature 458: 430-437.

28. Chin LS, Vavalle JP, Li L (2002) Staring, a novel E3 ubiquitin-protein ligase that targets syntaxin 1 for degradation. J Biol Chem 277: 35071-35079.

29. Wheeler TC, Chin LS, Li Y, Roudabush FL, Li L (2002) Regulation of synaptophysin degradation by mammalian homologues of seven in absentia. J Biol Chem 277: 10273-10282.

30. Ivanova D, Dirks A, Fejtova A (2016) Bassoon and piccolo regulate ubiquitination and link presynaptic molecular dynamics with activity-regulated gene expression. $J$ Physiol 594: 5441-5448.

31. Waites CL, Leal-Ortiz SA, Okerlund N, Dalke H, Fejtova A, et al. (2013) Bassoon and Piccolo maintain synapse integrity by regulating protein ubiquitination and degradation. EMBO J 32: 954-969.

32. Yao I, Takagi H, Ageta H, Kahyo T, Sato S, et al. (2007) SCRAPPER-dependent ubiquitination of active zone protein RIM1 regulates synaptic vesicle release. Cell 130: 943-957.

33. Tada H, Okano HJ, Takagi H, Shibata S, Yao I, et al. (2010) Fbxo45, a novel ubiquitin ligase, regulates synaptic activity. J Biol Chem 285: 3840-3849.

34. Lachance V, Degrandmaison J, Marois S, Robitaille M, Genier S, et al. (2014) Ubiquitylation and activation of a Rab GTPase is promoted by a beta(2)AR-HACE1 complex. J Cell Sci 127: 111-123.

35. Di Iorgi N, Allegri AE, Napoli F, Calcagno A, Calandra $\mathrm{E}$, et al. (2014) Central diabetes insipidus in children and young adults: etiological diagnosis and long-term outcome of idiopathic cases. J Clin Endocrinol Metab 99: 1264 1272 . 